\title{
Remote ischemic post-conditioning protects against myocardial ischemia/reperfusion injury by inhibiting the Rho-kinase signaling pathway
}

\author{
FENG MIN $^{1,2^{*}}$, XIAN JIE JIA ${ }^{1 *}$, QIN GAO $^{2,3}$, FANG NIU $^{1}$, ZHI YUAN HU ${ }^{2}$, \\ YA LING HAN ${ }^{1}$, HONG JIE SHI ${ }^{1}$ and YING YU ${ }^{2,3}$ \\ ${ }^{1}$ Department of Epidemiology and Statistics, ${ }^{2}$ Research Center of Bengbu Medical College, \\ ${ }^{3}$ Department of Physiology, Bengbu Medical College, Bengbu, Anhui 233030, P.R. China
}

Received November 14, 2018; Accepted October 11, 2019

DOI: $10.3892 /$ etm.2019.8176

\begin{abstract}
The aim of the present study was to observe the effect of Rho-kinase on remote ischemic post-conditioning (RIPostC) and explore the underlying mechanisms. Male Sprague Dawley rats $(n=32)$ were randomly distributed into four groups: Sham group, ischemia/reperfusion (I/R) group, RIPostC group and I/R with fasudil group (I/R+Fas). Infarction size was detected by triphenyltetrazolium chloride staining. The levels of creatine kinase (CK), lactate dehydrogenase (LDH), superoxide dismutase (SOD), malondialdehyde (MDA) and cardiac troponin I (cTnI) were measured using an ultraviolet spectrophotometer. The mRNA expression levels of Rho-associated coiled-coil containing protein kinase (ROCK)-1 and ROCK2, B-cell lymphoma 2 (Bcl-2) and $\mathrm{Bcl}-2$-associated $\mathrm{X}$ protein (Bax) were detected via reverse transcription-PCR. The protein expression levels of phosphorylated-myosin phosphatase target subunit (p-MYPT1) and phosphorylated-myosin light chain (p-MLC) were assessed by western blotting. The results demonstrated that RIPostC could decrease the infarct size, the levels of CK, LDH, cTnI and MDA and increase the activity of SOD compared with the I/R group. In addition, the mRNA expression of ROCK1 and ROCK2 was downregulated, the protein expression of p-MYPT1 and p-MLC was decreased, and the ratio of $\mathrm{Bcl}-2 / \mathrm{Bax}$ was elevated in the RIPostC groups compared with the I/R group. Notably, the aforementioned index in I/R with Fas group was similar to the RIPostC group and no significant difference was observed between RIPostC and I/R+Fas.
\end{abstract}

Correspondence to: Dr Ying Yu, Department of Physiology, Bengbu Medical College, 2600 Dong Hai Avenue, Bengbu, Anhui 233030, P.R. China

E-mail: yuying2011@126.com

*Contributed equally

Key words: remote ischemic postconditioning, rat, myocardium, ischemia/reperfusion injury, Rho-kinase, fasudil
These results revealed that RIPostC could attenuate I/R injury and the underlying mechanisms might be associated with a reduction in myocardial apoptosis and the suppression of the Rho-kinase signaling pathway.

\section{Introduction}

Coronary heart disease (CHD) is one of the leading causes of mortality worldwide, accounting for one-third of all mortalities worldwide each year (1). As a severe type of CHD, acute myocardial infarction (AMI) is the most common cause of mortality in China (2). The World Bank estimates that by 2030, 23 million Chinese patients will experience AMI annually (3). The most effective therapeutic measure against myocardial ischemia is to restore heart perfusion. However, the beneficial effects can be compromised by ischemia/reperfusion injury (I/R) (4). Myocardial I/R can induce further damage to the myocardium itself, leading to metabolic and functional disorders (5). The underlying mechanism of $I / R$ injury may be associated with oxidative stress, the inflammatory response, mitochondrial damage and cell apoptosis (6). In view of the dreaded complication of reperfusion and the heavy social burden, it is urgently necessary to further explore the mechanism of $\mathrm{I} / \mathrm{R}$ pathogenesis and develop effective intervention measures.

Remote ischemic post-conditioning (RIPostC), induced by several episodes of transient I/R intervention on distant tissues and organs away from the heart (such as the limbs, kidneys, small intestine and skeletal muscle), is as an effective strategy for myocardial protection against I/R injury (7). Due to its simple operation and significant curative effects, RIPostC can be performed noninvasively using a blood pressure cuff on the limb, and thus it has gained the attention of numerous researchers (8-10). RIPostC has been verified to have a beneficial effect in animal studies and randomized clinical trials (11-14). However, the underlying mechanism of RIPostC is still poorly understood (15).

Rho-associated coiled-coil containing protein kinase (ROCK), a serine/threonine kinase, has been identified as a downstream effector of RhoA. The ROCK isoforms, ROCK1 and ROCK2, were initially discovered as downstream targets of 
the small guanosine triphosphate-binding protein RhoA (16). The RhoA/Rho-kinase signaling pathway serves an important role in cardiovascular diseases including myocardial infarction, heart failure, hypertension and atherosclerosis (17). The Rho-kinase-mediated signaling pathway induces enhanced myocardial damage by mediating the phosphorylation of the downstream myosin light chain (MLC) and the myosin phosphatase target subunit (MYPT1) (18). Fasudil, a Rho-kinase inhibitor, has a beneficial effect on myocardial I/R by reducing myocardial infarct size, oxidative stress and cell apoptosis $(19,20)$. However, whether the cardioprotective effect on RIPostC is associated with downregulated Rho-kinase activity is unknown. Therefore, the present study was designed to investigate the effect of Rho-kinase on RIPostC and try to elucidate the underlying mechanism.

\section{Materials and methods}

Experimental animals. A total of 32 male Sprague-Dawley (SD) rats (weight, 250-300 g; age, 7-8 weeks), were purchased from the Laboratory Animal Center of Bengbu Medical College. The rats had free access to a normal diet and fresh water. All rats were kept at a constant temperature $\left(22-26^{\circ} \mathrm{C}\right)$ and humidity (50\%) with a 12-h light/dark cycle and were raised in individual cages. The animal research study protocol was following 'The Guide for the Care of Use of Laboratory Animals' published by the National Institute of Health and were approved by the Animal Use and Care Committee of Bengbu Medical College (21).

Materials and reagents. Fasudil was purchased from Tianjin Red Sun Pharmaceutical Co., Ltd., (cat. no. 1604071). Creatine kinase (CK; cat. no. 20170626), lactate dehydrogenase (LDH; cat. no. 20160606), superoxide dismutase (SOD; cat. no. 20160608) and malondialdehyde (MDA; cat. no. 20160620) assay kits were purchased from Nanjing Jiancheng Institute of Biotechnology. The cardiac troponin I (cTnI) ELISA kit was purchased from Calvin Biotechnology Co., Ltd., (cat. no. CK-E30258R). TRIzol ${ }^{\circledR}$ reagent was purchased from Invitrogen; Thermo Fisher Scientific, Inc. (cat. no. 15596026). RevertAid first strand cDNA synthesis kit was purchased from Thermo Fisher Scientific, Inc. (cat. no. 00398085). PCR master mix was purchased from Thermo Fisher Scientific, Inc. (cat. no. K0171). QuickBlock Blocking solution was purchased from Beyotime Institute of Biotechnology (cat. no. P0252). ROCK1, ROCK2, B-cell lymphoma 2 (Bcl-2), Bcl-2-associated X protein (Bax) and $\beta$-actin primers were purchased from Sangon Biotech Co., Ltd. The following primary antibodies were purchased from Cell Signaling Technology, Inc.: Mouse anti-phosphorylated (p)-MLC (cat. no. 3675), rabbit anti-MLC (cat. no. 3672), rabbit anti-p-MYPT1 (cat. no. 4563) and rabbit anti-MYPT1 (cat. no. 2634). Rabbit GAPDH antibody was purchased from Absin Bioscience Inc. (cat. no. JG26). Horseradish peroxidase (HRP)-linked anti-rabbit immunoglobulin ( $\mathrm{IgG}$; cat. no. BST11J12B54) and HRP-linked anti-mouse IgG (cat. no. BST11J12B50) were purchased from Wuhan Boster Biological Technology Co., Ltd. Immobilon western chemiluminescent HRP substrate (ECL) kit was acquired from EMD Millipore (cat. no. WBKLS0100).
Establishment of the myocardial I/R model. The rats were anesthetized by intraperitoneal injection of chloral hydrate (400 mg/kg) prior to the operation. Hemodynamic parameters and standard electrocardiograms (ECG) were continuously measured by the MedLab biological signal collection system to observe the changes of cardiac function in rats. Following tracheal intubation, ventilation was applied via respiratory equipment, with a respiratory rate of 70-80 times/min and a tidal volume of $20-30 \mathrm{ml} / \mathrm{kg}$ (22). Then, the chest was gently opened through the fourth and fifth ribs and the left anterior descending coronary artery (LAD) was identified. The LAD was ligated using a 5-0 silk (2 mm) suture. Additionally, a medical latex tube (socket inner diameter, $1.5 \mathrm{~mm}$ ) was placed between the ligature and the LAD. Myocardial ischemia was induced by tightening the ligature around the latex tube. Successful surgically induced myocardial ischemia was detected based on S-T segment elevation on an ECG (23). After $45 \mathrm{~min}$ of ischemia, the polyethylene tubes were loosened for $180 \mathrm{~min}$ to mimic reperfusion (24).

Experimental groups. A total of 32 healthy male SD rats were randomly divided into the following four groups $(n=8$ in each group): Sham group (Sham), I/R group (I/R), RIPostC group (RIPostC) and the I/R with fasudil group (I/R+Fas). In the Sham group, the LAD was threaded but not ligated for $225 \mathrm{~min}$ in vivo. In the I/R group, the LAD was ligated for $45 \mathrm{~min}$ (ischemia) followed by $180 \mathrm{~min}$ of the LAD open (reperfusion). In the RIPostC group, the same operation as the I/R group was performed; then following $15 \mathrm{~min}$ of ischemia, three cycles of right femoral artery clamping for $5 \mathrm{~min}$ and declamping for 5 min were conducted prior to reperfusion, as described in the authors previous study (24). In the I/R+Fas group, fasudil (10 mg/kg, a Rho-kinase inhibitor) was administered intravenously 5 min prior to myocardial reperfusion in I/R-treated rats (25).

Measurement of plasma $C K, L D H, M D A$ and SOD. At the end of the reperfusion period, carotid artery blood samples $(\sim 1.5 \mathrm{ml}$ per rat) were collected with disposable heparin blood vessels and centrifuged at $1,509 \mathrm{x}$ g for $15 \mathrm{~min}$ at $4^{\circ} \mathrm{C}$. The supernatant fluid was collected and stored at $-80^{\circ} \mathrm{C}$. The MDA contents and LDH, CK and SOD activities were measured by spectrophotometer at wavelengths of 532, 440,660 and $550 \mathrm{~nm}$, respectively according to the manufacturer's protocol.

Measurement of myocardial cTnI content. The cTnI content was measured using an ELISA kit at a wavelength of $450 \mathrm{~nm}$. The absorbances were measured and the contents were calculated according the manufacturer's protocol.

Assessment of myocardial infarct size. Infarct size was established by Evans blue and triphenyltetrazolium chloride (TTC) staining as described previously $(21,24)$. After the rats were sacrificed, the hearts were removed immediately and washed 2-3 times with PBS. Using re-occlusion of LAD in isolated Langendorff-perfused equipment, the hearts were perfused with $0.3 \mathrm{ml} 1 \%$ Evans blue dye to delineate the risk area. Following freezing at $-80^{\circ} \mathrm{C}$, the hearts were cut into $2 \mathrm{~mm}$ thick sections along the left ventricular transverse section and incubated with $1 \% \mathrm{TTC}$ at $37^{\circ} \mathrm{C}$ for $20 \mathrm{~min}$. Subsequently, the 
Table I. Reverse transcription-PCR primers for ROCK1, ROCK2, Bax, Bcl-2 and $\beta$-actin.

\begin{tabular}{lll}
\hline Gene & Primer & \multicolumn{1}{c}{ Sequence } \\
\hline ROCK1 & Forward & Product (bp) \\
& Reverse & 5'-GCA AAT GCG GGA GTT ACA AG-3' \\
ROCK2 & Forward & 5'-CAA GCC GAC TAA CGG TAT GAT C-3' \\
& Reverse & 5'-AGA ACC TGT CAA GCG TGG TAG TG-3' \\
Bax & Forward & 5'-GAC AGC CAT CCT TCT ATT CGT GA-3' \\
& Reverse & 5'-GGA TCG AGC AGA GAG GAT GG-3' \\
Bcl-2 & Forward & 5'-TGG TGA GTG AGG CAG TGA GG-3' \\
& Reverse & 5'-CTG GTG GAC AAC ATC GCT CTG-3' \\
$\beta$-actin & Forward & 5'-GGT CTG CTG ACC TCA CTT GTG-3' \\
& Reverse & 5'-CTG TAT GCC TCT GGT CGT AC-3' \\
\hline
\end{tabular}

ROCK1, Rho-associated coiled-coil containing protein kinase 1; ROCK2, Rho-associated coiled-coil containing protein kinase 2; Bax, Bcl-2-associated X protein; Bcl-2, B-cell lymphoma 2.

sections were fixed in $4 \%$ paraformaldehyde solution at $37^{\circ} \mathrm{C}$ for $30 \mathrm{~min}$. The gray area indicated the percentage infarct size (IS) and the red area represented the area at risk (AAR) was; these were quantified by computerized planimetry using ImageJ software (version 1.40; National Institutes of Health). Infarct size was calculated as the percentage of IS to AAR.

RNA extraction and semi-quantitative reverse transcriptionpolymerase chain reaction (RT-PCR) assay detecting ROCK1, ROCK2, Bax and Bcl-2 mRNA expression. Total RNA was extracted from the ischemic myocardial tissue using TRIzol ${ }^{\circledR}$ reagent. Total RNA ( $3 \mu \mathrm{g})$ was reverse transcribed into cDNA at $42^{\circ} \mathrm{C}$ and $1.5 \mu \mathrm{l} \mathrm{cDNA}$ was utilized for PCR amplification with the following temperature protocol: Denaturation at $95^{\circ} \mathrm{C}$ for $3 \mathrm{~min}$, followed by 40 cycles of denaturation at $95^{\circ} \mathrm{C}$ for $30 \mathrm{sec}$, annealing (ROCK $1,58^{\circ} \mathrm{C}$; ROCK2, $62.5^{\circ} \mathrm{C}$; Bax, $62.5^{\circ} \mathrm{C}$; $\mathrm{Bcl}-2,62.5^{\circ} \mathrm{C}$; and $\beta$-actin, $62.5^{\circ} \mathrm{C}$ ) for $30 \mathrm{sec}$, and extension at $72^{\circ} \mathrm{C}$ for $30 \mathrm{sec}$, then $72^{\circ} \mathrm{C}$ for $10 \mathrm{~min}$ and held at $4^{\circ} \mathrm{C}$. The primer sequences are presented in Table I. The PCR products were analyzed on a $1.2 \%$ agarose gel. The densitometry results for the ROCK1, ROCK2, Bcl-2 and Bax genes were compared with the corresponding $\beta$-actin levels to analyze the relative expression, and the $\mathrm{Bcl}-2 / \mathrm{Bax}$ ratio was calculated using a Tanon-3500 Gel imaging system (GIS 4.1.2).

Western blot analysis of the protein expression of $M L C$, $p-M L C, M Y P T 1$ and $p-M Y P T 1$. Left anterior myocardium tissues from each group were collected and homogenized in a lysis buffer, which contained $20 \mathrm{mmol} / \mathrm{l}$ Tris $(\mathrm{pH} 7.4)$, $150 \mathrm{mmol} / 1 \mathrm{NaCl}, 1 \mathrm{mmol} / 1$ EDTA, $1 \mathrm{mmol} / 1$ EGTA, $1 \%$ Triton $\mathrm{X}-100,0.1 \%$ SDS and $1 \%$ protease inhibitor cocktail. The homogenates were sonicated by $3-5$ cycles (30 sec sonication and $10 \mathrm{sec}$ rest) at a frequency of $25 \mathrm{kHz}$ and $60 \%$ amplitude before they were centrifuged at $12,000 \mathrm{x} \mathrm{g}$ for $30 \mathrm{~min}$ at $4^{\circ} \mathrm{C}$. The protein concentration was determined using a bicinchoninic acid protein assay kit (Beyotime Institute of Biotechnology) (24). Total protein (45 $\mu \mathrm{g})$ was separated by SDS-PAGE using a $10 \%$ separation gel and a $5 \%$ concentrated gel, and transferred to PVDF membranes. The membranes were blocked with QuickBlock ${ }^{\mathrm{TM}}$ Blocking solution at $37^{\circ} \mathrm{C}$ for $2 \mathrm{~h}$ and then were incubated with the primary antibodies at $4^{\circ} \mathrm{C}$ overnight. Immunoblots were performed using the following antibodies: Rabbit GAPDH antibody (1:4,000), rabbit MYPT antibody (1:500), rabbit p-MYPT1 antibody $(1: 2,000)$, rabbit MLC antibody (1:500) and mouse p-MLC antibody $(1: 2,000)$. All membranes were incubated for $1 \mathrm{~h}$ with the corresponding HRP-linked anti-rabbit $\operatorname{IgG}(1: 8,000)$ or HRP-linked anti-mouse IgG $(1: 4,000)$ secondary antibodies. The membranes were analyzed by an ECL system. Gray values of the target bands were analyzed using ImageJ software.

Statistical analysis. All values were expressed as the mean \pm standard deviation $(n=8)$. Statistical comparisons were performed by one-way analysis of variance and the Newman-Keuls test using SPSS 16.0 software (SPSS, Inc.). $\mathrm{P}<0.05$ was considered to indicate a statistically significant difference.

\section{Results}

Changes in plasma $C K, L D H, M D A$ and SOD levels in each group. Compared with the Sham group, the activities of CK and $\mathrm{LDH}$, and MDA contents were significantly increased in the $\mathrm{I} / \mathrm{R}$ group $(\mathrm{P}<0.01)$, while $\mathrm{SOD}$ activities were significantly decreased $(\mathrm{P}<0.01)$. Compared with the I/R group, the activities of CK and LDH, and MDA contents were significantly decreased $(\mathrm{P}<0.01)$, and the SOD activities were significantly elevated in the RIPostC and I/R+Fas groups $(\mathrm{P}<0.01)$. However, the levels of CK, LDH, MDA and SOD were no significant differences between the RIPostC and I/R+Fas groups (P>0.05; Fig. 1).

Changes in plasma cTnI levels in each group. Compared with the Sham group, the contents of cTnI were significantly increased in the $I / R$ group $(\mathrm{P}<0.01)$. Compared with the I/R group, the contents of cTnI were significantly decreased in the RIPostC and $\mathrm{I} / \mathrm{R}+\mathrm{Fas}$ groups $(\mathrm{P}<0.01)$. However, there were no significant differences in cTnI levels between the RIPostC and I/R+Fas groups ( $\mathrm{P}>0.05$; Fig. 2). 

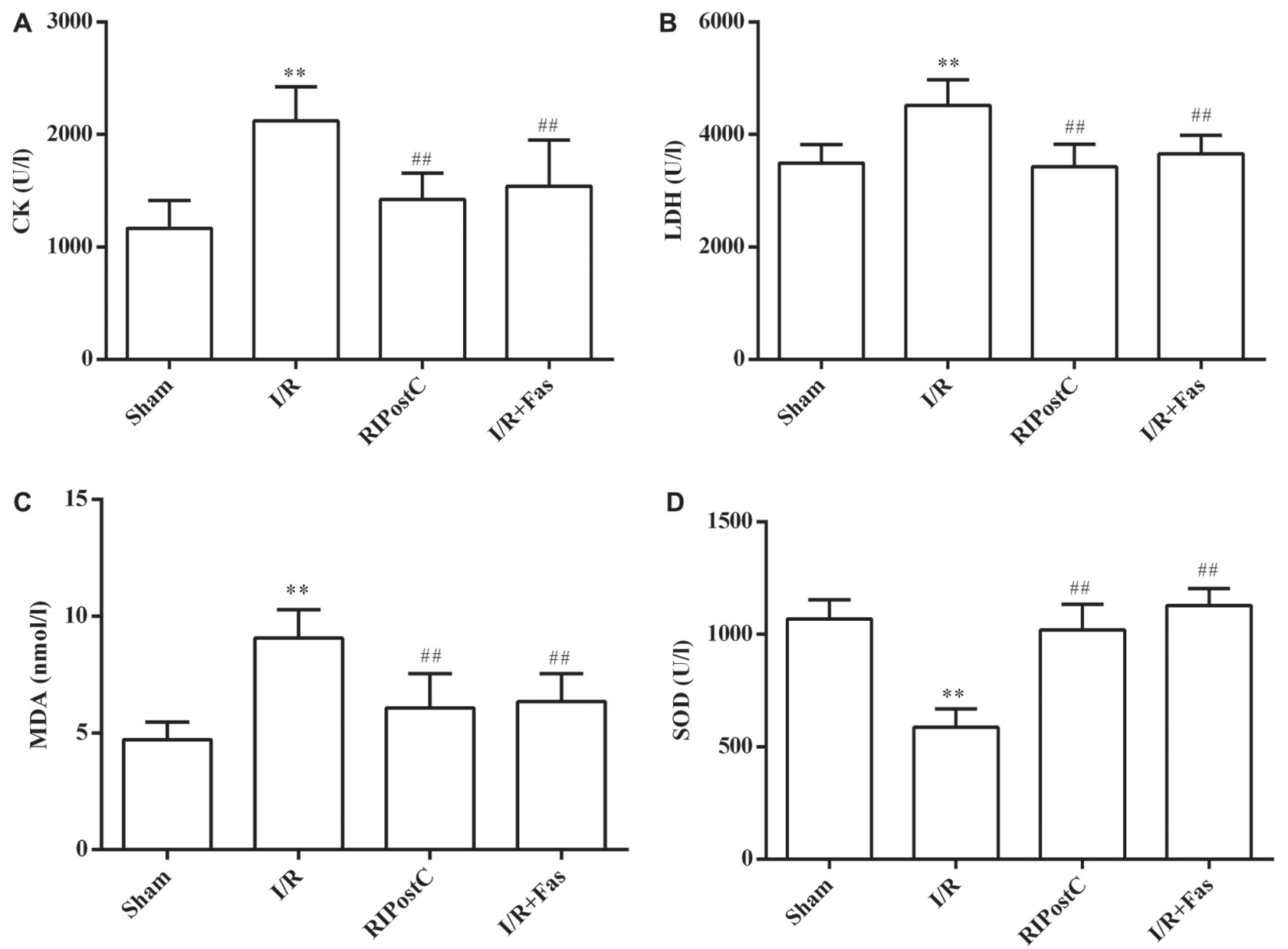

Figure 1. Changes to CK, LDH, MDA and SOD levels in the plasma. Effects of RIPostC and Rho-kinase on (A) CK activity, (B) LDH activity, (C) MDA content and (D) SOD activity, in the plasma of each groups. Values were expressed as the mean \pm standard deviation. ${ }^{* *} \mathrm{P}<0.01 \mathrm{vs}$. Sham group; ${ }^{\# \#} \mathrm{P}<0.01 \mathrm{vs}$. I/R group. I/R, ischemia/reperfusion; RIPostC, remote ischemic postconditioning; Fas, Fasudil; CK, creatine kinase; LDH, lactate dehydrogenase; MDA, malondialdehyde; SOD, superoxide dismutase.

Changes in myocardial infarct size in each group. Compared with the I/R group, the myocardial infarct size was significantly decreased in the RIPostC and $\mathrm{I} / \mathrm{R}+\mathrm{Fas}$ groups $(\mathrm{P}<0.01)$. There were no significant differences between the RIPostC and $\mathrm{I} / \mathrm{R}+$ Fas groups $(\mathrm{P}>0.05$; Fig. 3 ).

Expression of ROCK1, ROCK2, Bax and Bcl-2 mRNA in the myocardium. The RT-PCR results revealed that, compared with those in the Sham group, the mRNA expression of ROCK1 and ROCK2 was significantly increased $(\mathrm{P}<0.01)$, whereas the ratio of $\mathrm{Bcl}-2 / \mathrm{Bax}$ was significantly decreased in the $\mathrm{I} / \mathrm{R}$ group $(\mathrm{P}<0.01)$. However, compared with the $\mathrm{I} / \mathrm{R}$ group, the expression of ROCK1 and ROCK2 was significantly decreased, and the ratio of $\mathrm{Bcl}-2 / \mathrm{Bax}$ was increased in the RIPostC and $\mathrm{I} / \mathrm{R}+\mathrm{Fas}$ groups $(\mathrm{P}<0.01)$. In contrast to RIPostC group, the expression of ROCK1 and ROCK2, and the ratio of $\mathrm{Bcl}-2 / \mathrm{Bax}$ exhibited no statistical differences in the $\mathrm{I} / \mathrm{R}+\mathrm{Fas}$ group ( $\mathrm{P}>0.05$; Fig. 4).

Protein expression of $p-M L C, M L C, p-M Y P T 1$ and MYPT1. Compared with the Sham group, the protein expression of p-MLC and p-MYPT1 was significantly increased in the $\mathrm{I} / \mathrm{R}$ group $(\mathrm{P}<0.01)$. By contrast, the protein expression of p-MLC and p-MYPT1 in the RIPostC and I/R+Fas groups

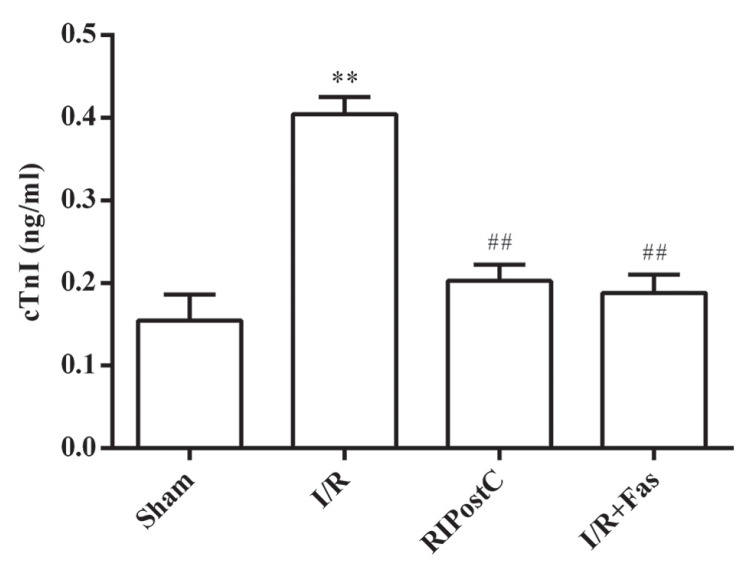

Figure 2. Effects of RIPostC and Rho-kinase on cTnI content in the plasma of each groups. Values were expressed as the mean \pm standard deviation. ${ }^{* *} \mathrm{P}<0.01$ vs. Sham group; ${ }^{\#} \mathrm{P}<0.01$ vs. I/R group. I/R, ischemia/reperfusion; RIPostC, remote ischemic postconditioning; Fas, Fasudil; cTnI, cardiac troponin I.

was decreased compared with the I/R group (P<0.01; Fig. 5). In contrast to those of the RIPostC group, there were no statistical differences in the I/R+Fas group ( $\mathrm{P}>0.05$; Fig. 4) 
A $\mathrm{I} / \mathrm{R}$

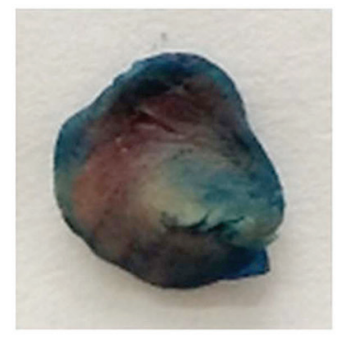

RIPostC

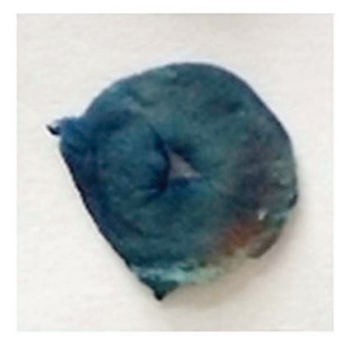

$\mathrm{I} / \mathrm{R}+\mathrm{Fas}$

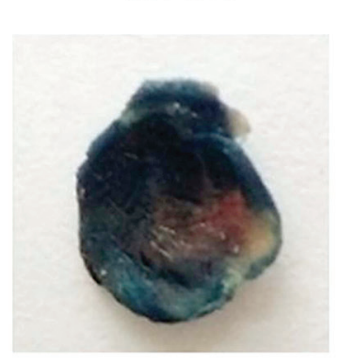

B

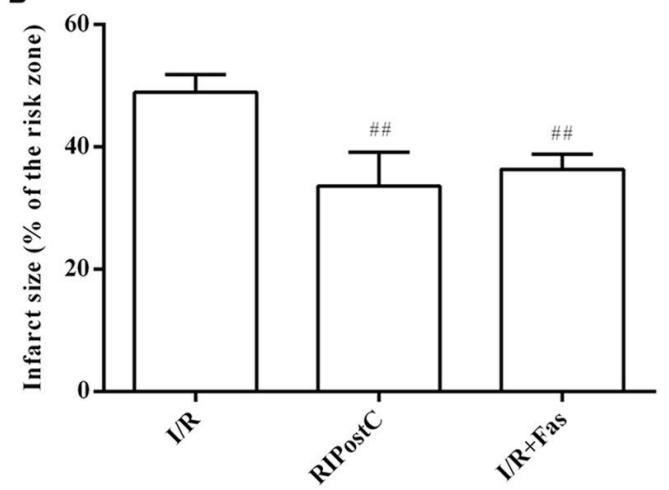

Figure 3. Alterations to myocardial infarct size in rats. (A) Representative images showing one stained section for each group and (B) comparison of infarct size in the rat myocardium of each group in vivo. Values are expressed as the mean \pm standard deviation. ${ }^{\# \#} \mathrm{P}<0.01$ vs. I/R group. I/R, ischemia/reperfusion; RIPostC, remote ischemic postconditioning; Fas, Fasudil.
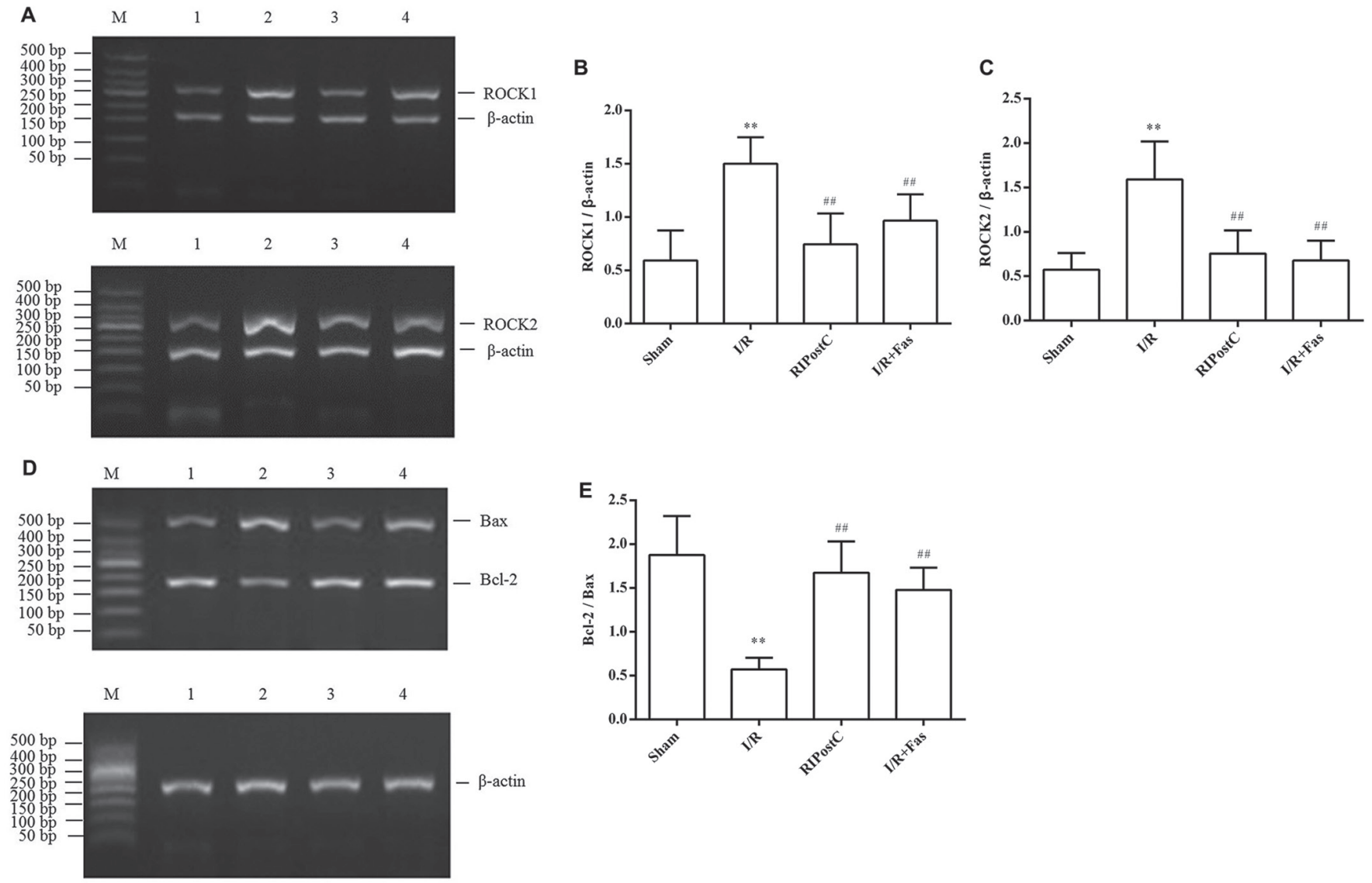

Figure 4. Changes in the expression levels of myocardial ROCK1, ROCK2, Bax and Bcl-2 at the mRNA level. Expression levels of myocardial (A) ROCK1 and ROCK2 mRNA, (B) quantification of the ROCK1/ $\beta$-actin, (C) quantification of the ROCK2/ $\beta$-actin, (D) Bax and Bcl-2 mRNA, and (E) quantification of the $\mathrm{Bcl}-2 / \mathrm{Bax}$ ratio for the different groups. Values were expressed as the mean \pm standard deviation. ${ }^{* *} \mathrm{P}<0.01 \mathrm{vs}$. Sham group; ${ }^{\# \#} \mathrm{P}<0.01 \mathrm{vs}$. I/R group. M, marker; 1, Sham; 2, I/R; 3, RIPostC; 4, I/R+Fas; I/R, ischemia/reperfusion; RIPostC, remote ischemic postconditioning; Fas, Fasudil; ROCK1, Rho-associated coiled-coil containing protein kinase 1 ; ROCK2, Rho-associated coiled-coil containing protein kinase 2; Bcl-2, B-cell lymphoma 2; Bax, Bcl-2-associated X protein.

\section{Discussion}

In the present study, it was demonstrated that RIPostC could provide protection against myocardial I/R injury. Rho-kinase pathway served an important role in myocardial $I / R$ injury. The present study also demonstrated that the Rho-kinase inhibitor fasudil produced attenuation of the myocardial infarction and myocardial apoptosis associated with I/R. It was further confirmed that RIPostC had a cardioprotective and anti-apoptotic effect through inhibition of the Rho-kinase signaling pathway.

RIPostC, which is induced by several episodes of brief $\mathrm{I} / \mathrm{R}$ in distant organs from the heart, has been developed as 
A

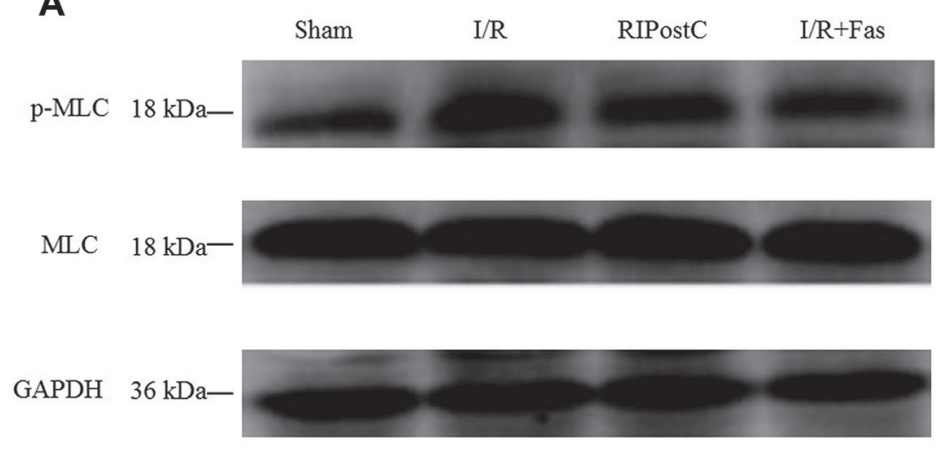

C

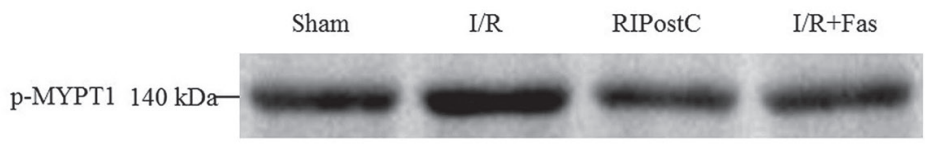

MYPT1 $140 \mathrm{kDa}$

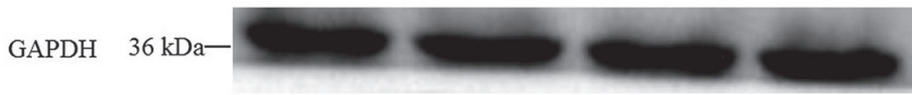

B
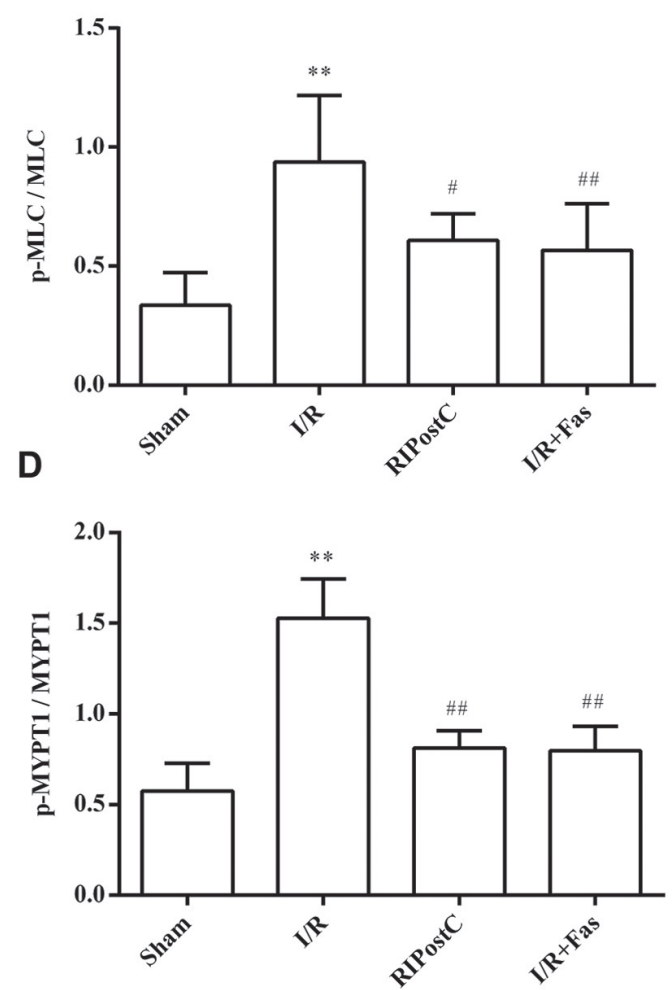

Figure 5. Changes in the expression levels of myocardial p-MLC, MLC, p-MYPT1 and MYPT1 at the protein level. Expression levels of (A and B) p-MLC and (C and D) p-MYPT1 in myocardial tissue. Representative western blots of (A) p-MLC and (C) p-MYPT1 in the myocardial tissue of each groups. $\beta$-actin was used as a loading control. Quantitative analysis of the (B) p-MLC/MLC and (D) p-MYPT1/MYPT1 protein expression ratios in the myocardium of rats. Values were expressed as the mean \pm standard deviation. ${ }^{* *} \mathrm{P}<0.01$ vs. Sham group; ${ }^{\#} \mathrm{P}<0.05$ and ${ }^{\# \#} \mathrm{P}<0.01 \mathrm{vs}$. I/R group. I/R, ischemia/reperfusion; RIPostC, remote ischemic postconditioning; Fas, Fasudil; p-MLC, phosphorylated-myosin light chain; MYPT1, phosphorylated-myosin phosphatase target subunit.

an effective strategy to protect against the harmful effects of I/R injury (26-29). Although the protective role has been widely recognized, the mechanism of the cardioprotective effect induced by RIPostC remains to be fully elucidated (15). Myocardial I/R injury can cause the generation of reactive oxygen species, calcium overload, the inflammatory response, mitochondrial damage, lipid peroxidation and further damage to the myocardial tissue (30). Myocardial infarction and the levels of LDH, CK and cTnI are frequently used to quantify the amount of myocardial damage $(31,32)$. In addition, measuring MDA content and SOD activity can reflect the degree of myocardial cell damage and the production of oxygen free radicals (33). The present study showed that RIPostC reduced the myocardial infarct size and inhibited the release of LDH, CK, cTnI and MDA, while increasing the activity of SOD. These results suggested that the RIPostC could effectively alleviate myocardial I/R injury. It was also demonstrated that the Rho-kinase inhibitor fasudil reduced infarct size, attenuated the increased levels of LDH, cTnI, CK and MDA, and reduced the activity of SOD, which had the cardioprotective effect similar to RIPostC. These observations indicated that inhibition of Rho-kinase and RIPostC could alleviate myocardial $\mathrm{I} / \mathrm{R}$ injury by reducing the production of free radicals.

Rho-kinase is a $160 \mathrm{kDa}$ serine/threonine protein kinase that acts as a downstream effector of the small $G$ protein RhoA. Rho-kinase has two highly homomeric isoforms, ROCK1 and ROCK2, which regulate cell formation, migration, proliferation and apoptosis (17). Both ROCK1 and
ROCK2 are expressed in vascular smooth muscle and the heart $(17,18)$. An increasing body of evidence has demonstrated that the Rho-kinase pathway serves an important role in myocardial I/R damage and inhibition of the Rho-kinase signaling pathway has beneficial effects on cardiac functions and apoptosis $(20,34)$. Apoptosis is an essential contributor to cardiac dysfunction (35). Bcl-2 family members serve important roles in regulating apoptotic signaling. The balance in the expression levels of the anti-apoptotic Bcl-2 and pro-apoptotic Bax proteins play a major role in the regulation of myocardial apoptotic cell death (24). Moreover, activation of Rho-kinase enhances the contractions of vascular smooth muscle cells, leading to coronary artery spasm and aggravating myocardial injury $(17,18)$. Certain studies have demonstrated that MLC and MYPT1 acted as the principal downstream effector protein of Rho-kinase, and their phosphorylation level could indirectly reflect the activity of Rho-kinase (36). Rho-kinase can increase MLC phosphorylation through direct effect on MLC or indirectly by inactivating MLC phosphatase (MYPT1) (37,38). Specifically, activation of Rho-kinase may increase calmodul in formation, upregulate the concentration of intracellular $\mathrm{Ca}^{2+}$ and induce the phosphorylation of MYPTl, which inhibits the activity of MLC phosphatase (MYPT1), causing MLC phosphorylation (37,39). In the present study, it was demonstrated that the expression of ROCK1 and ROCK2 were upregulated, the level of p-MLC and p-MYPT1 was increased, and the ratio of $\mathrm{Bcl}-2 / \mathrm{Bax}$ was decreased in the I/R group. However, the expression of ROCK1 and ROCK2 
were downregulated, the level of p-MLC and p-MYPT1 was decreased, and the ratio of $\mathrm{Bcl}-2 / \mathrm{Bax}$ was increased in the RIPostC group compared with the I/R group. Interestingly, the aforementioned index in the I/R with fasudil group was similar to RIPostC group and no significant difference was observed between RIPostC and I/R+Fas. The results revealed that RIPostC could downregulate the activity of Rho-kinase as well as inhibited the occurrence of myocardial apoptosis. These observations suggested that RIPostC could attenuate the I/R-induced injury in the myocardium by inhibiting the Rho-kinase signaling pathway through its anti-apoptotic effect.

There were several limitations in this study that are important to note. In the present study, it was only shown that the inhibition of the Rho-kinase was associated with RIPostC, but it was not investigated how RIPostC inhibits the Rho-kinase signaling pathway. Furthermore, the present results confirmed that RIPostC had a cardioprotective and anti-apoptotic effect through inhibition of the Rho-kinase signaling pathway, however, the study was not able to examine the Rho-kinase changes with pharmacological intervention. In addition, further analysis of cardiac function measurements and the direct evaluation of apoptosis are required to verify the cardioprotective effect of RIPostC by inhibiting the Rho-kinase pathway.

In conclusion, the present study demonstrated that RIPostC could reduce heart infarct size and cardiomyocyte apoptosis by reducing lipid peroxidation, suppressing oxidative stress and inhibiting Rho-kinase activity. Thus, the Rho-kinase signaling pathway may be an important mediator of RIPostC against myocardial I/R injury in vivo.

\section{Acknowledgements}

Not applicable.

\section{Funding}

The present study was supported by research grants from the Natural Science Foundation of China (grant no. 81770297), the Natural Science Foundation of Anhui Province (grant no. 1508085QH150), the Natural Science of the Education Department of Anhui Province (grant no. KJ2017A217), Outstanding Young Talents Support Program in Colleges and Universities of Anhui Province (grant nos. gxfxZD2016143 and gxfx2017062), and the National College Students Innovation Project (grant no. 201610367011), China.

\section{Availability of data and materials}

The datasets used and/or analyzed during the current study are available from the corresponding author on reasonable request.

\section{Authors' contributions}

FM, QG and YY designed the study, performed the experiments and drafted the manuscript. FN, ZYH, YLH and HJS performed the experiments and collated the experimental data. XJJ contributed to experiments, data interpretation and statistical analysis. All authors revised the manuscript critically for important intellectual content and gave the final approval of the version to be published.

\section{Ethics approval and consent to participate}

All experimental procedures were approved by the Institutional Animal Care and Use Committee of Bengbu Medical College of China (Bengbu, China).

\section{Patient consent for publication}

Not applicable.

\section{Competing interests}

The authors declare that they have no competing interests.

\section{References}

1. Anderson L, Oldridge N, Thompson DR, Zwisler AD, Rees K, Martin N and Taylor RS: Exercise-based cardiac rehabilitation for coronary heart disease: Cochrane systematic review and meta-analysis. J Am Coll Cardiol 67: 1-12, 2016.

2. Hu G, Yang P, Zeng Y, Zhang S and Song J: Danggui Buxue decoction promotes angiogenesis by up-regulation of VEGFR1/2 expressions and down-regulation of sVEGFR1/2 expression in myocardial infarction rat. J Chin Med Assoc 81: 37-46, 2018.

3. Jiang H, Wang H, Liu T, Yang Z, Zhang R and Han H: Co-cultured the MSCs and cardiomyocytes can promote the growth of cardiomyocytes. Cytotechnology 70: 793-806, 2018.

4. Matsumura K, Jeremy RW, Schaper J and Becher LC: Progression of myocardial necrosis during reperfusion of ischemic myocardium. Circulation 97: 795-804, 1998.

5. Buja LM and Entman ML: Modes of myocardial cell injury and cell death in ischemic heart disease. Circulation 98: 1355-1357, 1998.

6. Li X, Liu M, Sun R, Zeng Y, Chen S and Zhang P: Protective approaches against myocardial ischemia reperfusion injury. Exp Ther Med 12: 3823-3829, 2016.

7. Kerendi F, Kin H, Halkos ME, Jiang R, Zatta AJ, Zhao ZQ, Guyton RA and Vinten-Johansen J: Remote postconditioning. Brief renal ischemia and reperfusion applied before coronary artery reperfusion reduces myocardial infarct size via endogenous activation of adenosine receptors. Basic Res Cardiol 100: 404-412, 2005.

8. Hausenloy DJ and Yellon DM: Ischaemic conditioning and reperfusion injury. Nat Rev Cardiol 13: 193-209, 2016.

9. Huang J, Xu D, Guo Q, Ou B, Ling Q, Li J, Yang Z and Tang W: Remote ischemic postconditioning improves myocardial dysfunction via the risk and safe pathways in a rat model of severe hemorrhagic shock. Shock 49: 460-465, 2018.

10. Li CM, Zhang XH, Ma XJ and Luo M: Limb ischemic postconditioning protects myocardium from ischemia-reperfusion injury. Scand Cardiovasc J 40: 312-317, 2006.

11. Andreka G, Vertesaljai M, Szantho G, Font G, Piroth Z, Fontos G, Juhasz ED, Szekely L, Szelid Z, Turner MS, et al: Remote ischaemic postconditioning protects the heart during acute myocardial infarction in pigs. Heart 93: 749-752, 2007.

12. Deftereos S, Giannopoulos G, Tzalamouras V, Raisakis K, Kossyvakis C, Kaoukis A, Panagopoulou V, Karageorgiou S, Avramides D, Toutouzas K, et al: Renoprotective effect of remote ischemic post-Conditioning by intermittent balloon inflations in patients undergoing percutaneous coronary intervention. J Am Coll Cardiol 61: 1949-1955, 2013.

13. Dezfulian C, Garrett M and Gonzalez NR: Clinical application of preconditioning and postconditioning to achieve neuroprotection. Transl Stroke Res 4: 19-24, 2013.

14. Hu X, Lv T, Yang SF, Zhang XH and Miao YF: Limb remote ischemic post-conditioning reduces injury and improves long-term behavioral recovery in rats following subarachnoid hemorrhage: Possible involvement of the autophagic process. Mol Med Rep 17: 21-30, 2018. 
15. Aimo A, Borrelli C, Giannoni A, Pastormerlo LE, Barison A, Mirizzi G, Emdin M and Passino C: Cardioprotection by remote ischemic conditioning: Mechanisms and clinical evidences. World J Cardiol 7: 621-632, 2015.

16. Liao JK, Seto M and Noma K: Rho kinase (ROCK) inhibitors. J Cardiovasc Pharmacol 50: 17-24, 2007.

17. Satoh K, Fukumoto Y and Shimokawa H: Rho-kinase: Important new therapeutic target in cardiovascular diseases. Am J Physiol Heart Circ Physiol 301: H287-H296, 2011.

18. Shimokawa H, Sunamura $S$ and Satoh K: RhoA/Rho-kinase in the cardiovascular system. Circ Res 118: 352-366, 2016.

19. Ye HW, Fang TT, Gu XY, Wang Y, Zhu GY, Yu Y and Gao Q: Role of autophagy in fasudil-induced Rho kinase inhibition for protection against myocardial ischemia-reperfusion injury in rats. Nan Fang Yi Ke Da Xue Xue Bao 36: 1706-1711, 2016 (In Chinese)

20. Zhang J, Liu XB, Cheng C, Xu DL, Lu QH and Ji XP: Rho-kinase inhibition is involved in the activation of PI3-kinase/Akt during ischemic-preconditioning-ind-uced cardiomyocyte apoptosis. Int J Clin Exp Med 7: 4107-4114, 2014.

21. Zhang WP, Zong QF, Gao Q, Yu Y, Gu XY, Wang Y, Li ZH and Ge M: Effects of endomorphin-1 postconditioning on myocardial ischemia/reperfusion injury and myocardial cell apoptosis in a rat model. Mol Med Rep 14: 3992-3998, 2016.

22. Cheng XY, Gu XY, Gao Q, Zong QF, Li XH and Zhang Y: Effects of dexmedetomidine postconditioning on myocardial ischemia and the role of the PI3K/Akt-dependent signaling pathway in reperfusion injury. Mol Med Rep 14: 797-803, 2016.

23. Yang J, Fan Z, Yang J, Ding J, Yang C and Chen L: microRNA-22 attenuates myocardial ischemia-reperfusion injury via an anti-inflammatory mechanism in rats. Exp Ther Med 12: 3249-3255, 2016.

24. Yu Y, Jia XJ, Zong QF, Zhang GJ, Ye HW, Hu J, Gao Q and Guan SD: Remote ischemic postconditioning protects the heart by upregulating ALDH2 expression levels through the PI3K/Akt signaling pathway. Mol Med Rep 10: 536-542, 2014.

25. Li Y, Zhu W, Tao J, Xin P, Liu M, Li J and Wei M: Fasudil protects the heart against ischemia-reperfusion injury by attenuating endoplasmic reticulum stress and modulating SERCA activity: The differential role for PI3K/Akt and JAK2/STAT3 signaling pathways. PLos One 7: e48115, 2012.

26. Schmidt MR, Sloth AD, Johnsen J and Bøtker HE: Remote ischemic conditioning: The cardiologist's perspective. J Cardiovasc Med (Hagerstown) 13: 667-674, 2012.

27. Liem DA, Verdouw PD and Duncker DJ: Transient limb ischemia induces remote ischemic preconditioning in vivo. Circulation 107: e218-e219, 2003.
28. Roubille F, Franck-Miclo A, Covinhes A, Lafont C, Cransac F, Combes S, Vincent A, Fontanaud P, Sportouch-Dukhan C, Redt-Clouet C, et al: Delayed postconditioning in the mouse heart in vivo. Circulation 124: 1330-1336, 2011.

29. Xin P, Zhu W, Li J, Ma S, Wang L, Liu M, Li J, Wei M and Redington AN: Combined local ischemic postconditioning and remote perconditioning recapitulate cardioprotective effects of local ischemic preconditioning. Am J Physiol Heart Circ Physiol 298: H1819-H1831, 2010.

30. Kalogeris T, Bao Y and Korthuis RJ: Mitochondrial reactive oxygen species: A double edged sword in ischemia/reperfusion vs preconditioning. Redox Boil 2: 702-714, 2014.

31. Mythili S and Malathi N: Diagnostic markers of acute myocardial infarction. Biomed Rep 3: 743-748, 2015.

32. O'Brien PJ: Cardiac troponin is the most effective translational safety biomarker for myocardial injury in cardiotoxicity. Toxicology 245: 206-218, 2008.

33. Gao YH, Chen L, Ma YL and He QY: Chronic intermittent hypoxia aggravates cardiomyocyte apoptosis in rat ovariectomized model. Chin Med J (Engl) 125: 3087-3092, 2012.

34. Bian H, Zhou Y, Yu B, Shang D, Liu F, Li B and Qi J: Rho-kinase signaling pathway promotes the expression of PARP to accelerate cardiomyocyte apoptosis in ischemia/reperfusion. Mol Med Rep 16: 2002-2008, 2017.

35. Cheng Y, Tan J, Li H, Kong X, Liu Y, Guo R, Li G, Yang B and Pei M: Cardioprotective effects of total flavonoids from Jinhe Yangxin prescription by activating the PI3K/Akt signaling pathway in myocardial ischemia injury. Biomed Pharmacother 98: 308-317, 2018.

36. Jiao Y, Fan YF, Wang YL, Zhang JY, Chen S and Chen ZW: Protective effect and mechanism of total flavones from rhododendron simsii planch flower on cultured rat cardiomyocytes with anoxia and reoxygenation. Evid Based Complement Alternat Med 2015: 863531, 2015.

37. Shi J and Wei L: Rho kinase in the regulation of cell death and survival. Arch Immunol Ther Exp (Warsz) 55: 61-75, 2007.

38. Amano M, Ito M, Kimura K, Fukata Y, Chihara K, Nakano T, Matsuura Y and Kaibuchi K: Phosphorylation and activation of myosin by Rho-associated kinase (Rho-kinase). J Biol Chem 271: 20246-20249, 1996.

39. Wang Y, Wang X, Liu W and Zhang L: Role of the Rho/ROCK signaling pathway in the protective effects of fasudil against acute lung injury in septic rats. Mol Med Rep 18: 4486-4498, 2018.

This work is licensed under a Creative Commons Attribution-NonCommercial-NoDerivatives 4.0 International (CC BY-NC-ND 4.0) License. 